\title{
Prophylactic effect of ethyl pyruvate on renal ischemia/reperfusion injury in rats
}

\begin{abstract}
Background: Following our hypothesis that oxidative stress might play a primary role in renal ischemia/reperfusion injury (RIRI), we investigated if ethyl pyruvate (EPy) with potent antioxidant activity might prevent or alleviate RIRI induced in rats.

Methods: Sprague-Dawley rats were randomly divided into four groups: (A) Sham, (B) renal ischemia/reperfusion (RIR), (C) RIR with EPy supplement (RIR+EPy), and (D) RIR with Mann supplement (RIR+Mann). Mannitol (Mann), a preoperative agent being clinically used, was tested for comparison with EPy. Rats were subjected to 40-min ischemia, followed by 24-h reperfusion. Either EPy or Mann was given to rats 30 min prior to ischemia and immediately before the reperfusion period.

Results: The RIR and RIR+Mann groups showed palpable kidney injuries with the $\sim 5$-fold elevated blood urea nitrogen (BUN) and creatinine $(\mathrm{Cr})$ levels, indicating renal dysfunction. However, the kidneys in the RIR+EPy group appeared merely normal (similar to the Sham's) with the basal BUN/Cr levels, indicating normal renal function. No effects on histology, BUN or Cr were yet seen with Mann. Moreover, specific kidney injury markers were upregulated and oxidative stress was also $\sim 2.1$-fold severer in the RIR group, whereas little changes in those markers and oxidative stress were seen with EPy supplement (RIR+EPy).

Conclusions: Although oxidative stress feasibly plays a key role in RIRI, EPy with antioxidant activity is capable of protecting the kidneys from such an assault. Thus, EPy (not Mann) should be considered as an effective perioperative renoprotective agent that could be used clinically.
\end{abstract}

Keywords: ethyl pyruvate, renal ischemia/reperfusion injury, oxidative stress, rat kidneys
Volume 8 Issue 4 - 2020

\author{
Roger Yau, Jonathan Bloom, Jonathan \\ Wagmaister, Majid Eshghi, Muhammad \\ Choudhury, Sensuke Konno \\ Department of Urology, New York Medical College, USA
}

Correspondence: Sensuke Konno, Ph.D. Department of Urology, New York Medical College, BSB Room A03,Valhalla, NY I0595 USA, Tel 9|4-594-3745, Fax 914-594-4428, Email sensuke_konno@nymc.edu

Received: August 03, 2020 | Published: August 24, 2020
Abbreviations: MDCK, madin-darby canine kidney; RIRI, renal ischemia/reperfusion injury; EPy, ethyl pyruvate; RIR, renal ischemia/reperfusion; Mann, mannitol; BUN, blood urea nitrogen; $\mathrm{Cr}$, creatinine; ARF, acute renal failure; OXS, oxidative stress; ROS, reactive oxygen species; $\mathrm{H}_{2} \mathrm{O}_{2}$, hydrogen peroxide; $\mathrm{NaPy}$, sodium pyruvate; IACUC, Institutional Animal Care and Use Committee; LPO, lipid peroxidation; MDA, malondialdehyde; NGAL, neutrophilgelatinase-associated lipocalin; Kim-1, kidney injury molecule 1; CLU, clusterin; SD, standard deviation; ANOVA, analysis of variance; MAPK, mitogen-activated protein kinase; h, hour(s); min, minute(s).

\section{Introduction}

Renal ischemia/reperfusion injury (RIRI) results in significant postoperative morbidity and mortality, often accompanied by acute renal failure (ARF). ${ }^{1}$ At the cellular level, RIRI can cause profound alterations in morphology and function of the proximal tubular epithelial cells, such as intracellular oxygen deficiency, ATP and glucose depletion, and calcium elevation. ${ }^{2}$ To protect the kidneys from such RIRI during surgery or (kidney) transplant and in the immediate postoperative period, particularly the perioperative interventions using various pharmaceutical agents such as dopamine and its analogues, diuretics, calcium channel blockers, angiotensin-converting enzyme inhibitors, hydration fluids etc. have been routinely employed., ${ }^{3,4}$ However, the outcomes have not been yet satisfactory and establishing a better and improved modality is urgently demanded. Accordingly, we hypothesized that RIRI could be primarily attributed to oxidative stress $(O X S)$, i.e. generation of reactive oxygen species (ROS), ${ }^{5}$ so that certain antioxidants could effectively abolish OXS. In fact, our early study ${ }^{6}$ showed that ethyl pyruvate (EPy), a potent antioxidant, counteracted with $\mathrm{H}_{2} \mathrm{O}_{2}$, a typical OXS inducer, to protect MDCK renal cells from renal cell injury in vitro. Although $\mathrm{H}_{2} \mathrm{O}_{2}$ led to a drastic $90 \%$ reduction in MDCK cell viability (only 10\% viable), EPy was capable of maintaining $>90 \%$ cell viability under such $\mathrm{H}_{2} \mathrm{O}_{2}$-exerted OXS [Figure 1(A)]. In addition, the severity of OXS went up to $\sim 3.1$ times higher (than control's) by $\mathrm{H}_{2} \mathrm{O}_{2}$, but EPy diminished or sustained OXS to the basal level [Figure 1(B)], exhibiting its potent antioxidant activity. No such effects were yet seen with mannitol (Mann), which was being clinically used. ${ }^{7}$ Hence, EPy with antioxidant activity appears to significantly protect renal cells from OXS in vitro. This also suggests that renal cell injury, caused by ischemia/reperfusion during kidney surgery/transplant, could be more likely linked to OXS. Taken together, OXS appears to trigger and facilitate RIRI but certain antioxidant regimen may effectively prevent or alleviate it.

It should be noted a little more about EPy. Structurally, EPy is a simple aliphatic ester derived from sodium pyruvate $(\mathrm{NaPy}),{ }^{8}$ a commonly known as pyruvate. ${ }^{9}$ Once this pyruvate is dissolved in aqueous solution, it is rapidly converted to parapyruvate, which inhibits the conversion of $\propto$-ketoglutarate to succinyl coenzyme A in the TCA cycle. ${ }^{8,9}$ However, parapyruvate cannot act as antioxidant capable of scavenging free radicals either. This is the main drawback of limited clinical utility of pyruvate. In contrast, EPy is safer, more stable and potent than pyruvate and has been shown to have antioxidant activity ${ }^{10}$ Moreover, the prophylactic or beneficial effects of EPy have been also reported in various organ systems - it has been shown to reduce or alleviate ischemia/reperfusion injury and hemorrhagic or endotoxic shock in animals. ${ }^{10-12}$ Nevertheless, exactly how EPy with antioxidant activity would work under such conditions has not been fully understood and more studies are certainly required. 
In the meantime, it was tempting for us to investigate whether EPy might have a prophylactic effect on RIRI induced in rats (in vivo). EPy was intraperitoneally given to rats $30 \mathrm{~min}$ prior to ischemia induced by renal pedicle occlusion and also given prior to the 24-h reperfusion period. We then assessed the renoprotective effect of EPy following this ischemia/reperfusion period. We also explored the protective mechanism of EPy against RIRI by examining the status of OXS and specific biochemical/physiological parameters. More details and interesting findings are described and discussed herein.

\section{Methods}

\section{Animal study}

All procedures were performed with the approval of the Institutional Animal Care and Use Committee (IACUC) at New York Medical College (Valhalla, NY). Twenty Sprague-Dawley rats were randomly divided into four experimental groups ( $\mathrm{n}=5$ per group): Group A (Sham), Group B [renal ischemia/reperfusion (RIR)], Group C [RIR with EPy supplement (RIR+EPy)], and Group D [RIR with Mann supplement (RIR+Mann)]. Mann was tested for comparison with EPy. RIR was carried out by inducing ischemia through 40 min renal pedicle occlusion, followed by $24 \mathrm{~h}$ reperfusion. Either EPy (200 mg/kg) or Mann (200 mg/kg) was given intraperitoneally to rats $30 \mathrm{~min}$ prior to ischemia and immediately before the start of reperfusion. Following 24-h reperfusion, blood specimens were collected and analyzed for blood urea nitrogen (BUN) and creatinine $(\mathrm{Cr})$, while kidney specimens were surgically excised and subjected to histopathologic examination and biochemical analyses.

\section{$\mathrm{BUN} / \mathrm{Cr}$ tests and histopathologic examination}

Blood and kidney specimens harvested from rats were immediately sent to the commercial pathology laboratory (Antech Diagnostics, New Hyde Park, NY) for BUN/Cr tests and histopathologic examination, respectively. Histopathologic examination was performed by two independent veterinary pathologists and the pathology reports were sent to us separately. Similarly, the results of BUN/Cr tests on all 5 rats in each group were reported separately to us.

\section{Lipid peroxidation (LPO) assay}

Approximately $50 \mathrm{mg}$ each of rat kidney specimen was first homogenized using a tissue grinder (with $\sim 20$ strokes) and cell extracts were obtained by centrifugation at $4{ }^{\circ} \mathrm{C}$. Fifteen $\mu \mathrm{g}$ each of various cell extracts were subjected to LPO assay to assess the severity of OXS by measuring the amount of malondialdehyde (MDA) formed. MDA is an end product formed in the plasma membrane through $\mathrm{OXS},{ }^{13}$ indicating as the more MDA formed, the greater OXS. The detailed procedures are described in the vendor's protocol (ABCAM, Cambridge, MA), and the amount of MDA formed is expressed by $\mathrm{nmol} / \mathrm{mg}$ protein determined from the MDA standards.

\section{Western blot analysis}

Possible effects of RIR on three specific biomarkers for acute renal cell injury ${ }^{14}$ were analyzed in kidney specimens by Western blots. An equal amount of tissue homogenates $(10 \mu \mathrm{g})$ was first subjected to $10 \%$ SDS-polyacrylamide gel electrophoresis and transferred to a nitrocellulose membrane. The blot (membrane) was incubated with primary antibodies against three RIR biomarkers, neutrophilgelatinase-associated lipocalin (NGAL), kidney injury molecule 1 (Kim-1), and clusterin (CLU) ${ }^{14}$ (Santa Cruz Biotechnology, Santa Cruz, CA), for $90 \mathrm{~min}$, followed by 30-min incubation with appropriate secondary antibody conjugates. Specific immunoreactive protein bands were then detected by chemiluminescence following manufacturer's protocol (Kirkegaard and Perry Laboratories).

\section{Statistical analysis}

All data are presented as mean $\pm \mathrm{SD}$ (standard deviation), and statistical differences between groups were assessed with either oneway ANOVA or the unpaired Student's $t$ test. Values of $p<0.05$ are considered to indicate statistical significance.

\section{Results}

\section{Histopathologic examination on rat kidneys}

To corroborate our in vitro study [Figure 1(A,B)], we examined if EPy could indeed prevent or alleviate RIRI in the rat kidneys (in vivo). Rats divided into the four groups were first subjected to $40-$ min ischemia, followed by 24-h reperfusion, as described in Materials and Methods. At the end of reperfusion, blood and kidney specimens were collected for further analyses. Histopathologic examination revealed that compared to Sham kidneys [Figure 2(A)], a large area of tubular necrosis, vacuolization, and cast formation was observed in the kidneys of the RIR group [Figure 2(B)]. However, EPy-given rats under RIR showed a little histologic change in the RIR+EPy group [Figure 2(C)], similar to the Sham group. Unlike EPy, Mann had virtually no effects on the kidneys [Figure 2(D)] and looked similar to the RIR group. Thus, these results show that EPy is capable of protecting the rat kidneys (from being injured) during the RIR period.
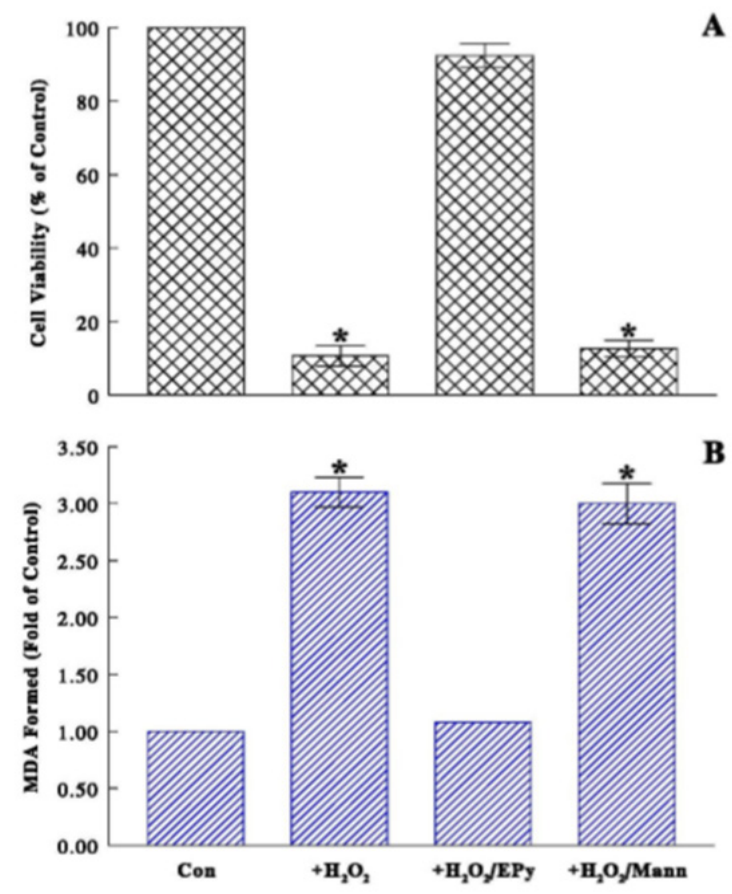

Figure I MDCK cells were treated with $\mathrm{H}_{2} \mathrm{O}_{2}(500 \mu M)$ alone or in combination with either EPy $(1 \mathrm{mM})$ or Mann $(10 \mathrm{mM})$ for $24 \mathrm{~h}$ for determining cell viability $(\mathbf{A})$ or $6 \mathrm{~h}$ for assessing severity of oxidative stress (B). Cell viability was expressed by the \% relative to controls (I00\%), while oxidative stress (as assessed by the amounts of MDA formed) was expressed by foldincrease relative to controls (I). All data are mean \pm SD (standard deviation) from three separate experiments $\left({ }^{*} \mathrm{p}<0.03\right.$ compared with control). 


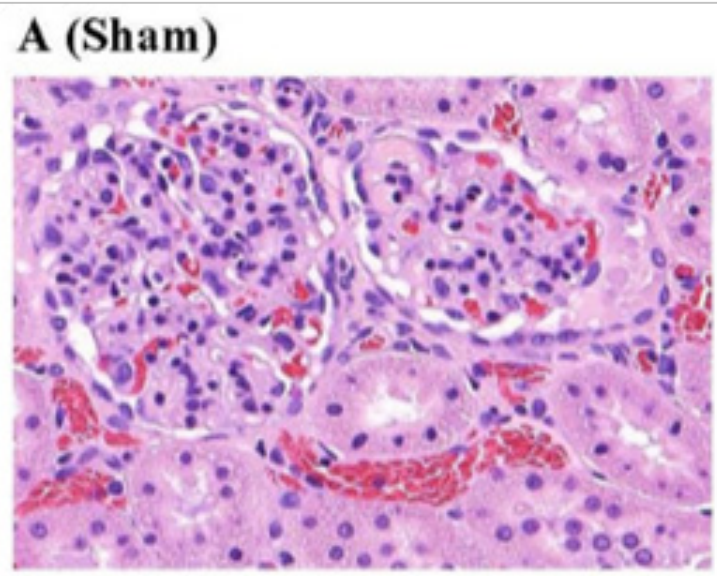

\section{B (RIR)}

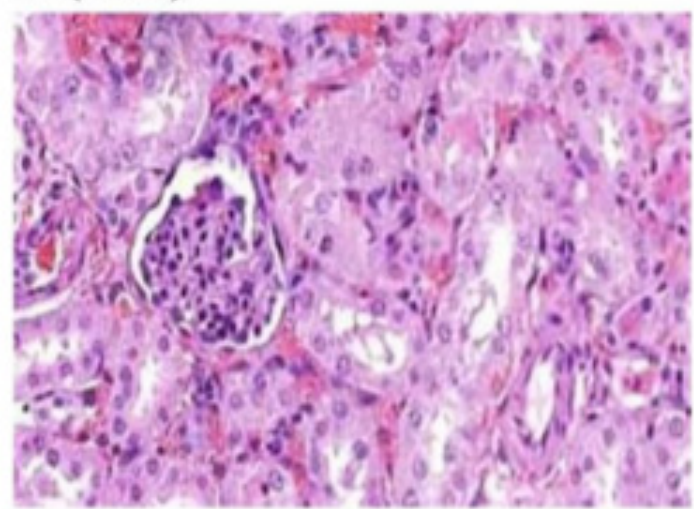

\section{C (RIR+EPy)}

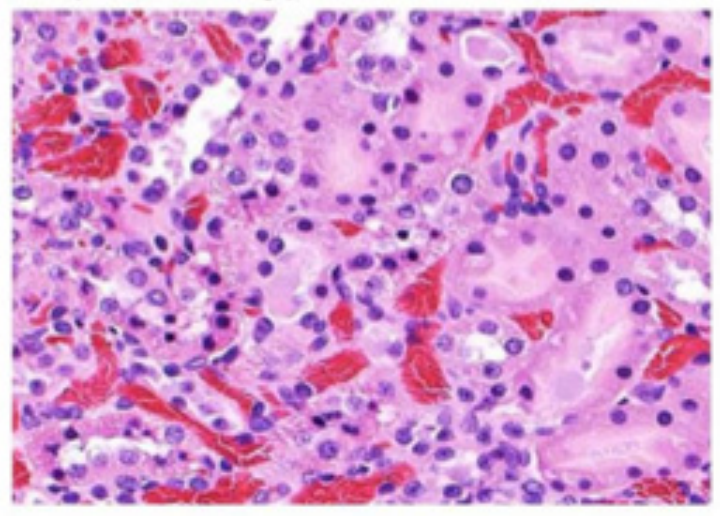

\section{D (RIR+Mann)}

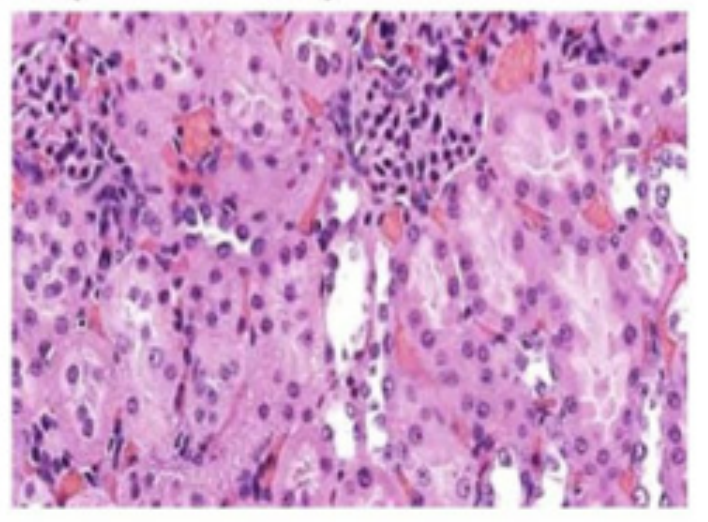

Figure 2 Histopathologic examination of kidney specimens. Four different experimental conditions of kidney specimens were histopathologically examined and photographed for the record. Those include: (A) Sham, (B) RIR, (C) RIR+EPy, and (D) RIR+Mann.

\section{Effects of EPy on renal function following RIR in rats}

Whether RIR-induced kidney injury would also affect renal function was assessed next. Blood analysis (Table 1) showed that the BUN level was profoundly ( $\sim 5$-fold) elevated in the RIR group. However, this elevated BUN was significantly decreased and close to that of the Sham group with EPy administration (RIR+EPy). Mann had little effects on the BUN level elevated under RIR, which remained $\sim 4.7$-fold higher than that in the Sham group. Similarly, the Cr level was a $\sim 3.5$-fold higher in the RIR group (than the Sham), but such elevated $\mathrm{Cr}$ also drastically declined with EPy, whereas Mann had little effects with the elevated $\mathrm{Cr}$ level (remaining $~ 3.1$-fold higher than the Sham). Thus, although RIR can cause renal dysfunction with elevating the BUN and Cr levels, EPy appears to effectively prevent/

Table I Effects of EPy on renal function under RIR reduce a loss of renal function under RIR.

\section{Exertion of RIR-induced OXS on kidney specimens}

We next performed LPO assay on kidney specimens to assess the involvement of OXS during RIR procedures. As shown in Figure 3 , the amount of MDA formed in the rat kidneys under RIR was a 2 -1-fold greater than that in the Sham group, indicating severe exertion of OXS on the kidneys. However, such severe OXS was significantly diminished with EPy administration (RIR+EPy), while Mann had little effects with the $\sim 1.8$-fold higher MDA level than the Sham. Thus, these results suggest that RIR-induced OXS could be effectively reduced with EPy owing to antioxidant activity. As Mann seems to have any (protective) effects against RIR, it was omitted from the rest of our study.

\begin{tabular}{lllll}
\hline & Sham & RIR & RIR+EPy & RIR+Mann \\
\hline BUN $(\mathrm{mg} / \mathrm{dl})$ & $17.32 \pm 2.02$ & $87.47 \pm 4.87^{*}$ & $19.77 \pm 1.72$ & $81.62 \pm 5.02^{*}$ \\
Creatinine $(\mathrm{mg} / \mathrm{dl})$ & $0.48 \pm 0.04$ & $1.69 \pm 0.11^{*}$ & $0.58 \pm 0.04$ & $1.50 \pm 0.09^{*}$
\end{tabular}

${ }^{*} \mathrm{p}<0.05$ compared with Sham (control).

$\mathrm{RIR}$, renal ischemia/reperfusion; EPy, ethyl pyruvate; Mann, mannitol; BUN, blood urea nitrogen 


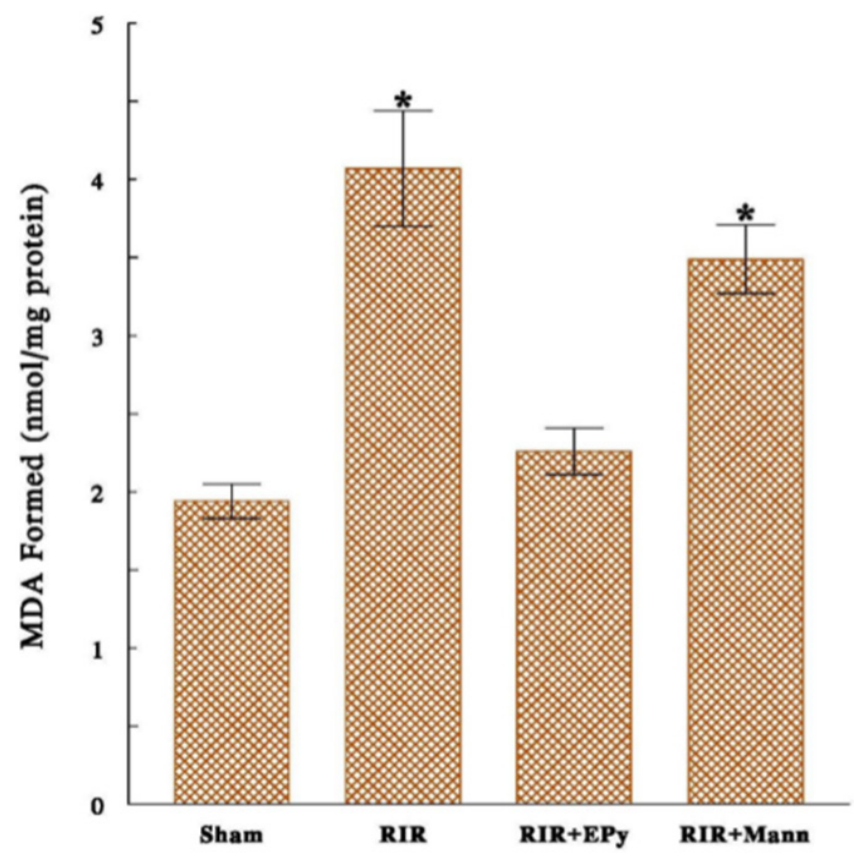

Figure 3 Exertion of oxidative stress. Whether the RIR procedures would exert oxidative stress was determined by the amounts of MDA formed in rat kidneys. Severity of oxidative stress in four experimental conditions was calculated and expressed as indicated. All data are mean \pm SD from three individual specimens of each experimental group $\left({ }^{*} p<0.05\right.$ compared with Shams).

\section{Effects of RIR or EPy on RIRI biomarkers in kidney specimens}

Lastly, how specific biomarkers for acute renal cell injury were affected by RIR with administration of EPy was analyzed in kidney specimens using Western blots. Expressions of three markers, NGAL, Kim-1, or CLU ${ }^{14}$ are shown in Figure 4. All three biomarkers were up-regulated or more intensely expressed under RIR (the RIR group), compared to those in the Sham group. However, rats supplemented with EPy under RIR (RIR+EPy) showed no such enhanced expressions, which looked similar to those in the Sham. The up-regulation of these markers further confirms apparent renal cell injury, ${ }^{14}$ which was yet prevented with EPy as all markers remained intact (RIR+EPy). This also demonstrates the renoprotective effect of EPy.

\section{Discussion}

It is now believed that RIRI could be primarily attributed to oxidative stress (OXS), ${ }^{5}$ implying its potential prevention with antioxidants. Nevertheless, there are many antioxidants available and we have to study and find out which one(s) is most effective on prevention of RIRI. We have then learned that ethyl pyruvate (EPy), a pyruvate derivative, had potent antioxidant activity. ${ }^{8,10}$ Hence, it was of our interest to investigate if EPy might prevent or reduce the incidence of RIRI in a rat model. Not only histopathologic examination but also physiological and biochemical analyses were performed to have a better understanding of RIRI and mode of action of EPy. Following 40-min ischemia/24-h reperfusion of the rat kidneys (RIR), histopathologic examination of the rat kidneys showed palpable cellular alterations with injury [Figure 2(B)]
However, those rats supplemented with EPy during RIR showed some marginal effects in their kidneys [Figure 2(C)] that yet looked similar to Shams [Figure 2(A)]. We also found that the serum levels of BUN and Cr have significantly elevated with RIR, indicating a loss of kidney function (Table 1). Nevertheless, such elevated BUN and $\mathrm{Cr}$ levels (with RIR) declined to nearly the same levels of Shams with EPy supplement (RIR+EPy) (Table 1). Therefore, these findings suggest that EPy may effectively protect the rat kidneys from RIRI and maintain normal renal function. In contrast, as Mann had no such protective effects against RIR, those kidneys were markedly injured with renal dysfunction.

To explore how RIRI is caused, we tested if OXS would be involved and play a primary role. LPO assay revealed that severe OXS was exerted on the rat kidneys by RIR, although it was reduced to nearly the Sham's level with EPy supplement (Figure 3). No such effects were yet seen with Mann supplement. Thus, EPy, not Mann, has potent antioxidant activity to significantly diminish detrimental OXS. It should be also noted that OXS has been shown to be a primary cause of various renal cell injury/damage including RIRI. ${ }^{5,15}$ In fact, those injuries by nephrotoxic agents and even by extracorporeal shock wave lithotripsy are also associated with OXS. ${ }^{16,17}$ It is then conceivable that many renal diseases/disorders resulted from renal cell injury are somehow linked to the certain degrees of OXS. To further confirm RIRI (through RIR), the status of three biomarkers for RIRI or acute renal cell injury were examined. Such study revealed that all three markers, NGAL, Kim-1, and CLU, ${ }^{14}$ were up-regulated following RIR (Figure 4). Particularly, NGAL has been shown to be elevated/induced under OXS or conditions of ischemia, ${ }^{18}$ providing cellular protection. Although its protective mechanism has not been fully understood, it has been reported that NGAL protection against RIRI was substantially attributed to the inhibition of renal tubular epithelial cell apoptosis through bcl-2/Bax signaling pathways. ${ }^{19}$ Additionally, Kim-1 expression is known to be highly elevated in both acute and chronic kidney injury and also believed to play a key role in macrophage activation via mitogen-activated protein kinase (MAPK) pathway in kidney disease. ${ }^{20}$ However, the specific functions of NGAL and Kim-1 yet remain to be fully elucidated. Nevertheless, since these three markers have been documented to be up-regulated (highly induced) when renal cells were injured or damaged, ${ }^{14}$ our finding here is indeed consistent with RIR-induced renal cell injury.

\section{Conclusion}

Oxidative stress may play a primary role in renal ischemia/ reperfusion injury as evidenced by this study. Such renal ischemia/ reperfusion can result in renal dysfunction, accompanied by the significantly elevated levels of serum blood urea nitrogen and creatinine in rats. Increased oxidative stress, mediated through renal ischemia/reperfusion, leads to severe renal cell injury, indicated by the up-regulation of three renal injury biomarkers (NGAL, Kim-1, and CLU). Nevertheless, ethyl pyruvate with potent antioxidant activity is capable of effectively protecting the kidneys (renal cells) from detrimental oxidative stress induced by renal ischemia/reperfusion. The rat kidneys remain nearly intact or have little effects with ethyl pyruvate during ischemia/reperfusion. Therefore, it is rather plausible that ethyl pyruvate (not mannitol) should be considered as an effective prophylactic agent against renal ischemia/reperfusion injury that is primarily induced by oxidative stress. It may have significant clinical implications as well. 


\section{$\begin{array}{lll}\text { Sham } & \text { RIR } & \text { RIR+EPy }\end{array}$}
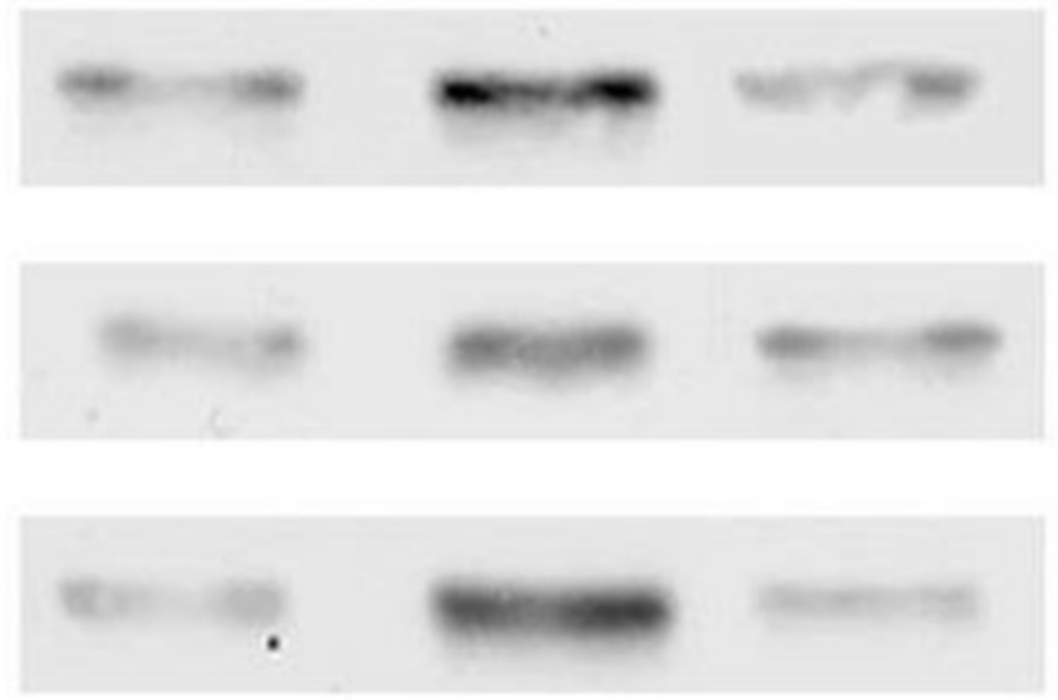

\section{$\beta$-actin}

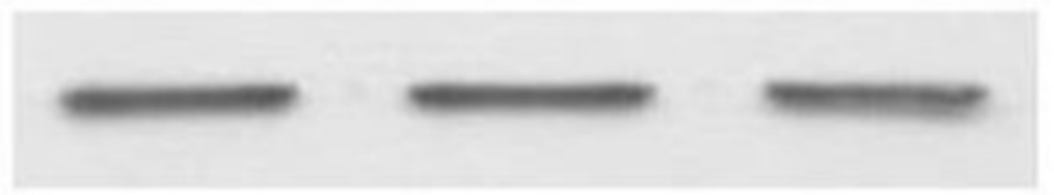

Figure 4 Up-regulation of RIRI biomarkers with RIR. Kidney specimens from three experimental conditions were analyzed for three RIRI biomarkers, such as NGAL, Kim-I, and CLU, using Western blots. Autoradiographs of these markers are shown and $\beta$-actin was also run as a protein loading control.

\section{Acknowledgements}

We are greatly indebted to Mr. Michio Tatsumi (Saraya International, Inc.) for his generous financial support for this study.

\section{Conflicts of interest}

The authors declare that there is no conflict of interest.

\section{References}

1. Versteilen AM, Di Maggio F, Leemreis JR, et al. Molecular mechanisms of acute renal failure following ischemia/reperfusion. Int J Artif Organs. 2004;27:1019-1029.

2. Padanilam BJ. Cell death induced by acute renal injury: a perspective on the contributions of apoptosis and necrosis. Am J Physiol Renal Physiol. 2003;284:F608-627.

3. Zacharias M, Mugawar M, Herbison GP, et al. Interventions for protecting renal function in the perioperative period. Cochrane Database Syst. 2013;9:CD003590.

4. Conger JD. Interventions in clinical acute renal failure: what are the data? Am J Kidney Dis. 1995;26:565-576.

5. Baliga R, Ueda N, Walker PD, et al. Oxidant mechanisms in toxic acute renal failure. Am J Kidney Dis. 1997;29:465-477.
6. Bloom J, Patel N, Wagmaister J, et al. Prophylactic effect of ethyl pyruvate on ischemia/reperfusion injury mediated through oxidative stress. Int Urol Nephrol. 2019;51:85-92.

7. Poullis M. Mannitol and cardiac surgery. Thorac Cardiovasc Surg. 1999;47)1):58-62.

8. Cruz RJ Jr, Harada T, Sasatomi E, et al. Effects of ethyl pyruvate and other $\alpha$-keto carboxylic acid derivatives in a rat model of multivisceral ischemia and reperfusion. $J$ Surg Res. 2011;165:151-157.

9. Fink MP. Ethyl pyruvate: a novel anti-inflammatory agent. Crit Care Med. 2003;31(Suppl 1):S51-S56.

10. Sims CA, Wattanasirichaigoon S, Menconi MJ, et al. Ringer's ethyl pyruvate solution ameliorates ischemia/reperfusion-induced intestinal mucosal injury in rats. Crit Care Med. 2001;29:1513-1518.

11. Uchiyama T, Delude RL, Fink MP. Dose-dependent effects of ethyl pyruvate in mice subjected to mesenteric ischemia and reperfusion. Intensive Care Med. 2003;29:2050-2058.

12. Tsung A, Kaizu T, Nakao A, et al. Ethyl pyruvate ameliorates liver ischemia-reperfusion injury by decreasing hepatic necrosis and apoptosis. Transplantation. 2005;79:196-204.

13. Dargel R. Lipid peroxidation: a common pathogenetic mechanism? Exp Toxic Pathol. 1992;44:169-181. 
14. Charlton JR, Portilla D, Okusa MD. A basic science view of acute kidney injury biomarkers. Nephrol Dial Transplant. 2014;29(7):13011311.

15. Baud L, Ardaillou R. Reactive oxygen species: production and role in the kidney. Am J Physiol. 1986;251:F765-776.

16. Diamond JR. The role of reactive oxygen species in animal models of glomerular disease. Am J Kidney Dis. 1992;19:292-300.

17. Andreoli SP, McAteer JA. Reactive oxygen molecule-mediated injury in endothelial and renal tubular epithelial cells in vitro. Kidney Int. 1990;38:785-794.
18. Roudkenar MH, Halabian R, Roushandeh AM, et al. Lipocalin 2 regulation by thermal stresses: protective role of Lcn2/NGAL against cold and heat stresses. Exp Cell Res. 2009;315(18):3140-3151.

19. An S, Zang X, Yuan W, et al. Neutrophil gelatinase-associated lipocalin (NGAL) may play a protective role against rats ischemia/reperfusion renal injury via inhibiting tubular epithelial cell apoptosis. Ren Fail. 2013;35(1):143-149.

20. Tian L, Shao X, Xie Y, et al. Kidney injury molecule-1 is elevated in nephropathy and mediates macrophage activation via the Mapk signaling pathway. Cell Physiol Biochem. 2017;41(2):769-783. 